-Editorial

\title{
A step forward in the understanding of prefrontal cortical functions
}

\author{
Bao-Ming $\mathrm{Li}^{1, *}$, Shintaro Funahashi ${ }^{2}$ \\ ${ }^{1}$ Institute of Life Science, Nanchang University, Nanchang 330031, China \\ ${ }^{2}$ Kokoro Research Center, Kyoto University, Kyoto 606-8501, Japan \\ *Guest Editor of the Special Issue. E-mail: bmli@ncu.edu.cn
}

(c) Shanghai Institutes for Biological Sciences, CAS and Springer-Verlag Berlin Heidelberg 2015

The prefrontal cortex (PFC) is an integrative area that guides behaviors and thoughts ${ }^{[1-3]}$. During the past century, a great many studies have been performed on PFC functions, using a variety of technical approaches such as anatomy, lesion, neuropsychology, electrophysiology, and functional imaging ${ }^{[2,3]}$. It is well known that the PFC plays an essential role in inhibitory control of behavior, regulation of attention, working memory, decision-making, and behavioral organization ${ }^{[1-6]}$.

In October 2013, the $4^{\text {th }}$ International Conference on Prefrontal Cortex was held in Nanchang University (Nanchang, China), co-organized by Bao-Ming Li (Nanchang University, China), Shintaro Funahashi (Kyoto University, Japan), Yong-Di Zhou (East China Normal University, China), and Satoru Otani (Ryotokuji University, Japan). A group of scientists from China, Japan, the USA, Germany, and Brazil comprehensively introduced their studies and extensively discussed progress in the understanding of PFC functions. This special issue covers the reviews and reports by the speakers at the conference.

Evolutionarily, the PFC is the last-emerged structure of the brain. It is unknown how it has evolved into such a complex system in humans. The mesocortical dopamine projection into the PFC is essential for its cognitive functions. Lee and Goto propose a hypothesis that the mesocortical dopamine projection is one of the biological substrates involved in PFC evolution, and that this evolutionary process may result in the emergence of psychiatric disorders such as schizophrenia ${ }^{[7]}$.

Working memory refers to the cognitive process of maintaining information for seconds for subsequent goal- directed actions. Persistent neuronal firing in the sensory cortex and the PFC during the delay period in a working memory task has been considered to be the neuronal substrate for working memory maintenance. However, the role of the delay activity in the sensory cortex is thought to differ from that in the PFC. Ku et al. propose that the delay activities in the sensory cortex and the PFC reflect the quality and quantity of representations in working memory, respectively ${ }^{[8]}$.

In addition to maintaining working memory information, the PFC is thought to store memory traces for "rules" or "strategies" that determine the temporal structure of behavior, and this kind of memory might be served by synaptic plasticity in the PFC. Otani et al. review the induction of long-term synaptic plasticity in the medial PFC of rats, with special emphasis on the regulation of synaptic plasticity by dopamine. They conclude that synaptic plasticity in the PFC is powerfully modulated by dopamine in an inverted-U-shaped, dose-dependent manner ${ }^{[9]}$.

Cognitive disorders such as schizophrenia are associated with dysfunction of the PFC, and with changes in $\mathrm{N}$-methyl- $D$-aspartate receptors (NMDARs). The dorsolateral PFC, which is highly evolved in primates, subserves the higher cognitive functions especially affected in mental disorders such as schizophrenia. Wang and Arnsten review new evidence that demonstrates a key role of NR2B-containing NMDARs in the cognitive functions of the dorsolateral PFC, especially spatial working memory ${ }^{[10]}$.

Imaging techniques such as functional magnetic resonance imaging, positron emission tomography, and encephalography have been widely used to reveal 
the changes in brain activity associated with functions. However, it is difficult to draw conclusion about the causal relationships between them. Non-invasive electrical stimulation with direct or alternating currents allows the manipulation of brain activity and excitability and helps to uncover the causal relationships between brain activities and functions. Kuo and Nitsche review the principal mechanism of this approach and its application in exploring PFC functions ${ }^{[11]}$.

It is hypothesized that schizophrenia results from disrupted brain connectivity. It is important to know whether there is anatomical and functional dysconnectivity between the PFC and other brain regions, and how such dysconnectivity is linked to schizophrenia. Imaging techniques, such as diffusion tensor imaging and functional magnetic resonance imaging, make it possible to explore these issues. Zhou et al. review the recent progress in understanding the anatomical and functional dysconnectivity of the PFC in humans and their implications in schizophrenia ${ }^{[12]}$.

Much is understood about the interactions between cognitive functions, from language to cognitive control, from attention to memory. However, it has been difficult to dissociate these functions because their representative cortical regions are located in close proximity. Cai and Van der Haegen review a series of studies investigating the relationship between language and other cognitive functions by examining their functional lateralization. The authors argue that the hemispheric lateralization of language and its link with handedness could offer an appropriate starting point to shed light on the relationships between different cognitive functions ${ }^{[13]}$.

It is known that there is a close relationship between PFC dysfunctions and the symptoms of schizophrenia. In addition to typical features such as hallucinations, delusions, and withdrawal from social activities, schizophrenic patients present with many forms of cognitive disorders. For many years, there has been no suitable non-human primate model of schizophrenia. Mao et al. report a monkey model of this disease, which is induced by treatment with phencyclidine, a non-specific competitive NMDAR antagonist, and the symptoms of schizophrenia can be ameliorated by atypical antipsychotic drugs such as clozapine $^{[14]}$.
Attention deficit and hyperactivity disorder (ADHD) is a syndrome characterized by inattention, impulsivity, and/or hyperactivity, and seriously affects the cognitive development of children. The lack of suitable animal models limits the development of new medications for ADHD. Ma et al. review the previous studies in nonhuman primates showing that blockade of PFC $\alpha 2 A$ adrenoceptors mimics the major symptoms of ADHD, providing new insights for developing novel animal models, and contributing to the understanding of the neurobiological basis of $A D H D^{[15]}$.

This special issue also includes a Research Highlight ${ }^{[16]}$, introducing the work published in Science by Chengyu Li's laboratory at the Institute of Neuroscience, Chinese Academy of Sciences ${ }^{[17]}$. As noted above, working memory involves a short-term interval of retention termed the 'delay period'. It is known that neurons in the medial PFC of rodents (thought to be homologous to the dorsolateral PFC in monkeys) demonstrate elevated delayperiod activity, but its functional significance is unclear. Li's group optogenetically suppressed or enhanced the delay activity of pyramidal neurons in the medial PFC of mice. They found that behavioral performance was impaired during the learning stage of the working memory task (olfactory delayed-nonmatch-to-sample go/no-go task), but not after the mice were well-trained, indicating that the delay-period medial PFC activity is involved in learning the task, but not in the maintenance of working memory information. They conclude that properly-regulated delay-period medial PFC activity is critical for information retention when mice are learning the working memory task, but not for working memory maintenance after the animals are well-trained ${ }^{[17]}$.

We anticipate that this special issue will provoke further studies, providing a better understanding of the cognitive functions of the prefrontal cortex, and eventually lead to more effective treatments of prefrontal cortical cognitive disorders.

\section{REFERENCES}

[1] Goldman-Rakic PS. Circuitry of primate prefrontal cortex and regulation of behavior by representational memory. In: Plum F (Ed.). Handbook of Physiology: The Nervous System: Higher Functions of the Brain, Vol. 5, Sect. 1. Bethesda, MD: American Physiological Society, 1987: 373-417. 
[2] Fuster JM. The Prefrontal Cortex. 4th ed. New York: Academic Press, 2008.

[3] Luria AR. Higher Cortical Functions in Man. New York: Basic Books, 1966.

[4] Funahashi S, Bruce CJ, Goldman-Rakic PS. Mnemonic coding of visual space in the monkey's dorsolateral prefrontal cortex. J Neurophysiol 1989, 61: 331-349.

[5] Fuster JM, Alexander GE. Neuron activity related to shortterm memory. Science 1971, 173: 652-654.

[6] Miller EK, Erickson CA, Desimone R. Neural mechanisms of visual working memory in prefrontal cortex of the macaque. J Neurosci 1996, 16: 5154-5167.

[7] Lee YA, Goto Y. Prefrontal cortical dopamine from an evolutionary perspective. Neurosci Bull 2015, 31: 164-174.

[8] Ku YX, Bodner M, Zhou YD. Prefrontal cortex and sensory cortices during working memory: quantity and quality. Neurosci Bull 2015, 31: 175-182.

[9] Otani S, Bai J, Blot K. Dopaminergic modulation of synaptic plasticity in rat prefrontal neurons. Neurosci Bull 2015, 31: 183-190.

[10] Wang M, Arnsten AFT. Contribution of NMDA receptors to dorsolateral prefrontal cortical networks in primates. Neurosci
Bull 2015, 31: 191-197.

[11] Kuo MF, Nitsche MA. Exploring prefrontal cortex functions in healthy humans by transcranial electrical stimulation. Neurosci Bull 2015, 31: 198-206.

[12] Zhou Y, Fan L, Qiu C, Jiang T. Prefrontal cortex and the dysconnectivity hypothesis of schizophrenia. Neurosci Bull 2015, 31: 207-219.

[13] Cai Q, Van der Haegen L. What can atypical language hemispheric specialization tell us about cognitive functions? Neurosci Bull 2015, 31: 220-226.

[14] Mao P, Cui D, Zhao XD, Ma YY. Prefrontal dysfunction and a monkey model of schizophrenia. Neurosci Bull 2015, 31: 235-241.

[15] Ma CL, Sun X, Luo F, Li BM. Prefrontal cortical $\alpha_{2 A^{-}}$ adrenoceptors and a possible primate model of attention deficit and hyperactivity disorder. Neurosci Bull 2015, 31: 227-234.

[16] Xu N. Learn to memorize: shedding new light on prefrontal functions. Neurosci Bull 2015, 31: 242-244.

[17] Liu D, Gu X, Zhu J, Zhang X, Han Z, Yan W, et al. Medial prefrontal activity during delay period contributes to learning of a working memory task. Science 2014, 346: 458-463. 